Gefässchirurgie 2019 · 24:448-449

https://doi.org/10.1007/s00772-019-00564-9

(c) Springer Medizin Verlag GmbH, ein Teil von Springer Nature 2019

Sehr geehrte Leserinnen und Leser,

im klinischen Alltag beschäftigen uns Gefäßmediziner oft ganz andere Dinge als die, für die wir einmal Arzt geworden sind. Vor allem anderen sind wir jedoch dem Wohle des Patienten verpflichtet, der seine Gesundheit in unsere Hände legt.

Wir leben zunehmend in einem Spannungsfeld zwischen medizinischen, ökonomischen und ethischen Anforderungen, denen wir in unseren täglichen Entscheidungen jeweils gerecht werden wollen und sollen. Dass wir in medizinischer und ökonomischer Verantwortung als Ärzte stehen, ist uns bewusst. Unsere Therapien müssen auf eine wissenschaftlich begründete, patientenzentrierte und ressourcenbewusste Versorgung abzielen.

Die aktuelle gesundheitsökonomische Struktur bedingt einen erheblichen finanziellen Druck auf die Handelnden und schafft zugleich falsche ökonomische Anreize. Wir kennen die Berichte über die zunehmende Leistungsverdichtung (Stichwort Liegedauer) und den Personalabbau in den Krankenhäusern. Und wir machen unsere eigenen leidvollen Erfahrungen hiermit. Dies kann auf die Leistungsvergütung im DRGSystem zurückgeführt werden, in dem insbesondere Anreize zu Fallzahlerhöhungen und zur Zergliederung von komplexen Fällen in günstiger vergütete Einzelfälle bestehen. Es werden technische Leistungen ungleich höher vergütet als solche, die mit Gespräch, Zuhören und Empathie zu tun haben. Vor allem (elektive) operative Eingriffe triggern im DRG-System die Vergütung, während andere (konservative) Therapien oftmals schlecht vergütet werden oder gar nicht erlösrelevant sind. Auch die

\title{
T. Nowak
}

Klinik für Gefäßchirurgie und Angiologie, Alfried Krupp Krankenhaus Rüttenscheid, Essen, Deutschland

\section{Ethik und Ökonomie in der Gefäßmedizin}

interdisziplinäre bzw. interprofessionelle Abstimmung, die in unserem Fachgebiet eine zentrale Bedeutung besitzt, ist weitgehend „unvergütet“ [1]. Hierin liegt eine große Gefahr, dass Operationsindikationen „aufgeweicht“ werden. Es steht zu befürchten, dass der häufig vorhandene Ermessensspielraum, wann eine Operation durchgeführt werden muss, eher zugunsten des Krankenhauses als zugunsten der Patienten ausgelegt wird. An dieser Stelle darf Prof. Dr. med. Giovanni Maio (Institut für Ethik und Geschichte der Medizin, Universität Freiburg) zitiert werden: „Die medizinische Indikation ist ein Kernstück der ärztlichen Identität. Mit ihr bringt der Arzt zum Ausdruck, dass er dem Patienten nur das empfehlen wird, was für ihn, den Patienten, im Prinzip zuträglich ist. " [2]

Längst mehren sich die Stimmen, die von einer betriebswirtschaftlich motivierten Überversorgung insbesondere im Bereich elektiver Operationen berichten. Uns allen sind die Berichte über die im europäischen Vergleich überhohen Fallzahlen an Herzkatheteruntersuchungen, Knie-, Hüft- und Bandscheibenoperationen im Ohr. Aber wie sieht es in unserer eigenen Gefäßmedizin aus?

\section{) Die Zielsetzung ärztlichen Handelns darf nicht in ein ökonomisch orientiertes Handeln umdefiniert werden}

In manchen medizinischen Bereichen nehmen Beteiligte deutliche Anreize für Leistungsänderungen von Krankenhäusern wahr zugunsten lukrativerer Leistungen („Portfolioänderungen“,
„Rosinenpicken“) mit zum Teil fraglicher Indikationsstellung. Als Folge könnten geringer vergütete Leistungen ggf. nicht mehr erbracht werden und die Strategie der Krankenhäuser auf das Erzielen hoher Fallzahlen besser vergüteter Leistungen ausgerichtet werden [1]. Einem solchen Prozess müssen wir entschieden entgegentreten. Die Zielsetzung ärztlichen Handelns darf nicht in ein ökonomisch orientiertes Handeln umdefiniert werden (Bundesärztekammer in einer Stellungnahme [3]).

Um diese teils kaum auflösbaren Konflikte zeichnet sich eine gesellschaftliche und politische Diskussion mit widersprüchlichen Forderungen ab.

In der aktuellen gesundheitspolitischen Diskussion hat die BertelsmannStiftung mit einer Studie zur zukunftsfähigen Krankenhausversorgung eine bessere medizinische Versorgung in Deutschland nur bei Schließung von $50 \%$ der bestehenden Krankenhäuser für möglich erachtet. Naturgemäß wird dies sehr unterschiedlich bewertet. Und Gesundheitsminister Jens Spahn greift mit seinem Pflegepersonal-Stärkungsgesetz tief in den Ablauf der Akutkrankenhäuser ein. Eine wesentliche Nebenwirkung der angeordneten gesundheitspolitischen Therapie ist der passagere Abbau zahlreicher Krankenhausbetten im Intensiv- und Normalpflegebereich. Ist zu befürchten, dass dies manche medizinische Einrichtung auf längere Frist in die Knie zwingen kann? Jedenfalls steigt der Druck auf jeden einzelnen Medizinversorger im stationären Bereich an.

Aus der Kommission für konservative Gefäßtherapie der Deutschen Gesellschaft für Gefäßchirurgie und Gefäßmedizin heraus haben wir es für notwendig gehalten, ein Themenheft zu konzipieren, 
welches auf das alltägliche Spannungsfeld zwischen medizinischem Anspruch und Anforderungen und Ethik und Ökonomie in der Gefäßmedizin eingeht und uns einen Spiegel für das eigene Handeln vor Augen hält. Hier wollten wir sowohl Ärzte als auch Ökonomen und Ethiker zu Wort kommen lassen.

In unserem gefäßchirurgischen Kerngeschäft, der Therapie der pAVK IIb, lässt sich exemplarisch dieses Spannungsfeld zwischen Patientenorientierung, „wunscherfüllender Medizin“ und wirtschaftlicher Verantwortung vor dem Hintergrund sogenannter weicher Indikationen gut darstellen. Herr Hinrich Böhner zeigt diese Konflikte auf und beschreibt den wichtigen Aspekt der Patientenperspektive.

In der prophylaktischen Gefäßchirurgie zeigt sich ebenfalls das Spannungsfeld zwischen dem medizinisch Machbaren und dem ethisch begründeten Verzicht auf eine Maximaltherapie. Herr Marko Aleksic zeigt vor dem Hintergrund der aktuellen Literatur (bzw. der fehlenden Datenlage) auf, dass die Wahl des Prozederes eine ureigene persönliche Entscheidung ist und mit größtem Feingefühl von uns Gefäßmedizinern getroffen werden muss.

Dass medizinische Entscheidungsfindungen im Behandlungsalltag auch jenseits jedes betriebswirtschaftlichen Konfliktes sehr fordernd sein können, kann jeder Gefäßchirurg sicherlich bestätigen. Seit Längerem haben sich Ethikkommissionen in den Krankenhäusern etabliert und sich als förderlich zum Wohle des $\mathrm{Pa}$ tienten sowie als hilfreich für das Behandlungsteam gezeigt. Wie dieses Instrument praktisch angewendet werden kann, beschreiben Frau Tanja Löbbing und Herr Klaus Kobert sehr eindrücklich.

In diesem Zusammenhang ist auch die Sichtweise erfolgreicher Ökonomen im Gesundheitswesen wichtig. Wie beschrieben haben wir die Ökonomisierung in der Medizin zu verzeichnen, es fragt sich nur, wie diese gegenläufigen Interessen von Patient, Arzt und Ökonom zusammenpassen. Herr Rudolf Mintrop skizziert seinen persönlichen Weg als Geschäftsführer eines Maximalversorgers im Ruhrgebiet und beschreibt die strategischen Unterschiede zwischen
Non-Profit- und For-Profit-Kliniken aus seiner Sicht.

Trotz aller Herausforderungen und Spannungsfelder müssen wir in erster Linie Ärzte bleiben und unserer Verantwortung gegenüber dem Patienten gerecht werden. Ethiker und Ökonomen können uns hierbei helfen, die Medizin $\mathrm{zu}$ gestalten, die wir alle für uns selber gerne hätten.

Wir Ärzte haben die Verantwortung, dass wir nicht als willfährige Dienstleister im Gesundheitssystem agieren, sondern uns dem Patienten verpflichten. Denn wir sind in allererster Linie Mediziner und als leitende Ärzte nur nachgeordnet in ökonomischer Verantwortung. Unabhängig davon, wo wir im persönlichen Arbeitsumfeld der Patientenversorgung mit dem Thema Ökonomisierung der Medizin konfrontiert werden: Entscheidend ist, dass wir uns hiermit intensiv auseinandersetzen.

Ich wünsche eine gute Lektüre.

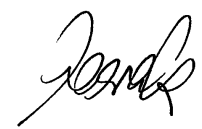

Dr. med. Thomas Nowak

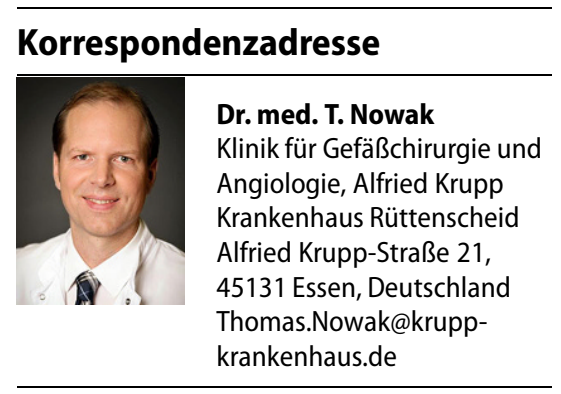

Interessenkonflikt. T. Nowak gibt an, dass kein Interessenkonflikt besteht.

\section{Literatur}

1. AWMF (2018) Stellungnahme: Medizin und Ökonomie - Maßnahmen für eine wissenschaftlich begründete, patientenzentrierte und ressourcenbewusste Versorgung

2. Klinkhammer G (2015) Indikation als Kernstück der ärztlichen Identität. Dtsch Arztebl 112:33-34

3. Bundesärztekammer (2015) Medizinische Indikationsstellung und Ökonomisierung. https://www.bundesaerztekammer. de/fileadmin/user_upload/downloads/pdfOrdner/Stellungnahmen/Stn_Medizinische_ Indikationsstellung_und_OEkonomisierung.pdf (Stellungnahme) Zugriffam 19.08.2019
Moderate Werte bei Blutdruck und Cholesterin verlängern das Leben

Dramatisch gesenkt wird das Risiko für Herz- und Gefäßerkrankungen, wenn Blutdruck- und Cholesterin-Werte lebenslang moderat gehalten werden. Das ist das Ergebnis einer aktuellen Studie an der Universität Cambridge.

Obwohl Herzinfarkte und Schlaganfälle durch anhaltend niedrige Werte bei Blutdruck und LDL-Cholesterin weitgehend vermieden werden können, stellen diese bei Frauen und Männern die häufigste Todesursache dar. Eine neue Studie zeigt nun, dass schon eine geringe, aber dauerhafte Senkung von systolischem Blutdruck und LDL-Cholesterin das kardiovaskuläre Erkrankungsrisiko hochsignifikant senkt. Ausgewertet wurden die Daten von über 400000 Briten. Die Teilnehmer waren durchschnittlich 65,2 Jahre alt; 54 Prozent waren Frauen. Bemerkenswertes Ergebnis der Studie: Werden lebenslang der LDLCholesterinspiegel um $39 \mathrm{mg} / \mathrm{dl}$ ( $1 \mathrm{mmol} / \mathrm{l})$ und gleichzeitig der systolische Blutdrucks um $10 \mathrm{mmHg}$ gesenkt, ergibt sich ein um 80 Prozent niedrigeres Risiko für kardiovaskuläre Erkrankungen und ein um 68 Prozent niedrigeres Risiko für einen kardiovaskulär verursachten Tod. Schon bei dauerhafter Abnahme des Cholesterins um $15 \mathrm{mg} / \mathrm{dl}$ und des Blutdrucks um $5 \mathrm{mmHg}$ halbiert sich das Erkrankungsrisiko.

„Der Knackpunkt ist, dass die Werte lebenslang niedrig sein müssen", so Prof. Laufs. „Da viele Patienten älter sind, haben Blutdruck und Cholesterin schon ein halbes Jahrhundert auf die Innenwände der Gefäße eingewirkt. Von Bedeutung ist rechtzeitig zu messen und vor allem rechtzeitig Maßnahmen zur Blutdruckund Cholesterin-Kontrolle zu ergreifen. Hierzu gehören an erster Stelle körperliche Aktivität und das Nicht-Rauchen, manchmal auch Medikamente." Vielleicht könnte schon im Kindes- und Jugendalter bei den sogenannten U-Untersuchungen auch auf diese Werte geachtet werden.

Quelle: Universitätsklinikum Leipzig AöR, http://www.uniklinik-leipzig.de/ 